\title{
OUH
}

Odense

Universitetshospital

\section{Three year follow-up after rehabiliation - a randomised study among radiated prostate cancer patients}

Karin B. Dieperink, Christoffer Johansen, Steinbjørn Hansen, Lis Wagner, Klaus K. Andersen, Lisbeth R. Minet, Olfred Hansen

\section{Purpose:}

- to study long-term effects of a multidisciplinary rehabilitation for prostate cancer (PCa) patients

- to study status adverse effects and quality of life (QoL) at different time points

\section{Methods:}

PCa patients treated with androgen deprivation therapy and radiotherapy $\mathrm{n}=161$ were randomized to:

a) two nurse lead psychosocial support sessions followed by two instructive sessions by a physical therapist $(n=79)$ or

b) usual care $(n=82)$

The primary outcome was irritative urinary sumscore (EPIC-26). In this follow-up study all participants reported disease-specific QoL (EPIC-26) and general QoL (SF-12) three years after radiotherapy.

Descriptive analysis and linear regression analysis adjusted for baseline and six month scores were conducted.
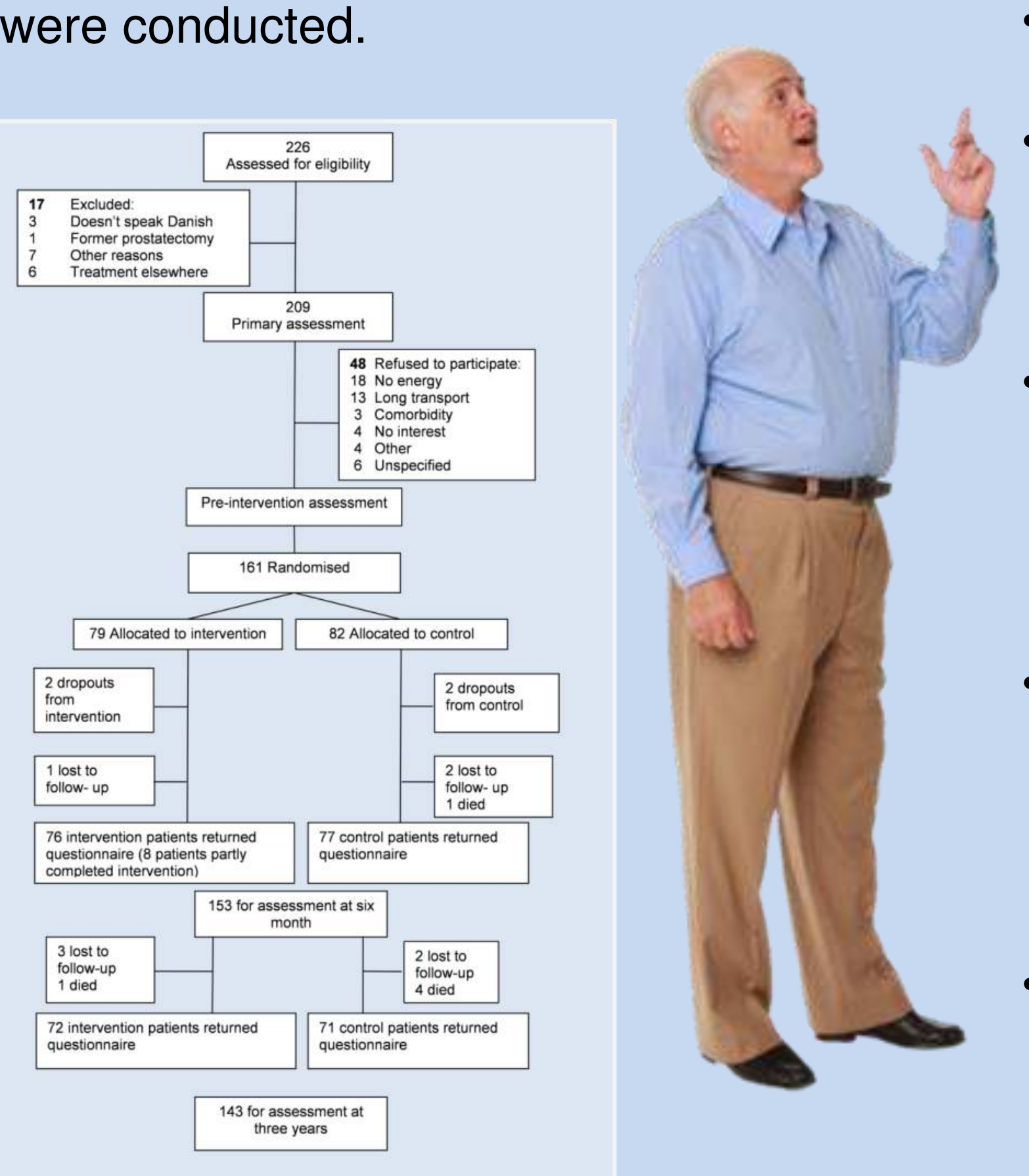

\section{Conclusions:}

We did not find a long-term effect of multidisciplinary rehabilitation in irradiated PCa patients. However, the intervention may positively influence bowel QoL

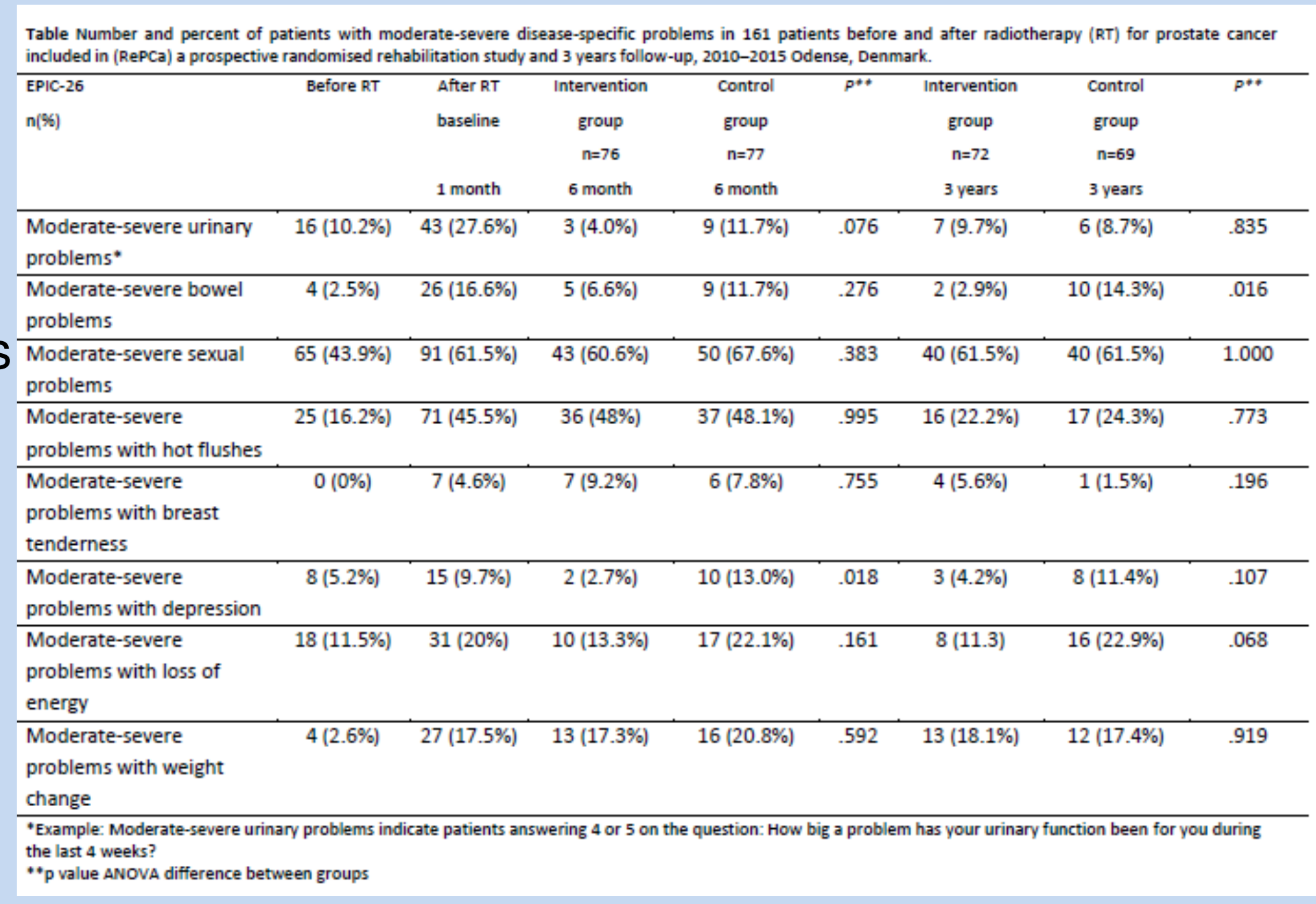

\section{Results:}

- $143 / 155$ (92\%) responded the questionnaire

- Three years after radiotherapy none of the EPIC domains were significantly different between groups

- Patients in the intervention group showed a trend for better irritative urinary sum score (0-100) with 86.12 SD 13.3 compared to 82.90 SD 15.0 in controls $P=.069$

- Significantly more patients in the control group had moderate to severe bowel problems 10 $(14.3 \%)$ compared to the intervention group 2 (2.9\%) $P=.016$

- $43(60 \%)$ patients in the intervention group reported the intervention useful 